\title{
Uncertainties in pentose-phosphate pathway flux assessment underestimate its contribution to neuronal glucose consumption: relevance for neurodegeneration and aging
}

\author{
Anne-Karine Bouzier-Sore ${ }^{1 *}$ and Juan P. Bolaños ${ }^{2 *}$ \\ ${ }^{1}$ Centre RMSB, Bordeaux Cedex, France, ${ }^{2}$ Institute of Functional Biology and Genomics, University of Salamanca-CSIC, \\ Salamanca, Spain
}

Keywords: astrocyte-neuron interactions, pentose-phosphate pathway, glycolysis, aging neuroscience, ${ }^{13} \mathrm{C}-\mathrm{NMR}$ spectroscopy

\section{OPEN ACCESS}

Edited by:

Enrique Cadenas,

University of Southern California, USA

Reviewed by:

Sebastian Cerdan,

Instituto de Investigaciones

Biomedicas Alberto Sols, Spain

João Laranjinha,

University of Coimbra, Portugal

${ }^{*}$ Correspondence:

Anne-Karine Bouzier-Sore, akb@rmsb.u-bordeaux2.fr Juan P. Bolaños, jbolanos@usal.es

Received: 25 March 2015 Accepted: 01 May 2015 Published: 19 May 2015

Citation: Bouzier-Sore $A-K$ and Bolaños JP (2015) Uncertainties in pentose-phosphate pathway flux assessment underestimate its contribution to neuronal glucose

consumption: relevance for neurodegeneration and aging.

Front. Aging Neurosci. 7:89. doi: 10.3389/fnagi.2015.00089

\section{The Impact of PPP in Redox and Energy Conservation}

The pentose-phosphate pathway (PPP) promotes the oxidative decarboxylation of glucose-6phosphate (G6P) in two consecutive steps, catalyzed by glucose-6-phosphate dehydrogenase (G6PD) and 6-phosphogluconate dehydrogenase (6PGD), yielding ribulose-5-phosphate (Ru5P) (Figure 1) (Wamelink et al., 2008). These steps constitute the so-called oxidative PPP branch, where the redox energy of G6P is conserved as $\operatorname{NADPH}\left(\mathrm{H}^{+}\right)$. Together with other $\operatorname{NADPH}\left(\mathrm{H}^{+}\right)$ regenerating systems, such as NADP-dependent isocitrate dehydrogenase and malic enzyme (ME), the oxidative PPP branch represents the most important source of reducing equivalents for (i) antioxidant enzymes, such as glutathione peroxidases and thioredoxin reductases, and (ii) fatty acid synthase (Dringen et al., 2007). Ru5P is isomerized into ribose-5-phosphate (R5P), which serves either as the precursor for nucleotide biosynthesis, or it continues metabolism through the non-oxidative PPP branch. In the latter, R5P epimerize into xylulose-5-phosphate (Xu5P), with which it transketolases producing sedoheptulose-7-phosphate (S7P) plus glyceraldehyde-3phosphate (G3P). In turn, S7P and G3P transaldolase to form fructose-6-phosphate (F6P) and erythrose-4-phosphate (E4P). E4P is then transketolated with Xu5P forming F6P and G3P. Thus, through the PPP, three moles of G6P yield three $\mathrm{CO}_{2}$, two F6P, and one G3P. Since PPP-derived F6P and G3P are glycolytic intermediates too, they can follow conversion into pyruvate. Thus, glycolysis and PPP are two different pathways that share common pools of F6P and G3P intermediates. Accordingly, G6P converted into pyruvate through the PPP conserves both the redox and the energetic values of glucose, highlighting a yet unrecognized high impact of PPP flux activity in redox/energy conservation.

Abbreviations: E4P, erythrose-4-phosphate; F6P, fructose-6-phosphate; F6B, fructose-1,6-bisphosphate; G3P, glyceraldehyde3-phosphate; G6P, glucose-6-phosphate; R5P, ribose-5-phosphate; G6PD, glucose-6-phosphate dehydrogenase; GSH, glutathione, reduced form; GSSG, glutathione, oxidized form; F26BP, fructose-2,6-bisphosphate; ME, malic enzyme; PEPCK, phosphoenolpyruvate carboxykinase; PFK1, 6-phosphofructo-1-kinase; PFKFB3, 6-phosphofructo-2-kinase/fructose-2,6bisphosphatase, isoform 3; PGI, phosphoglucose isomerase; 6PGD, 6-phosphogluconate dehydrogenase; PPP, pentosephosphate pathway; Ru5P, ribulose-5-phosphate; TCA, tricarboxylic acid; S7P, sedoheptulose-7-phosphate; Xu5P, xilulose5-phosphate. 


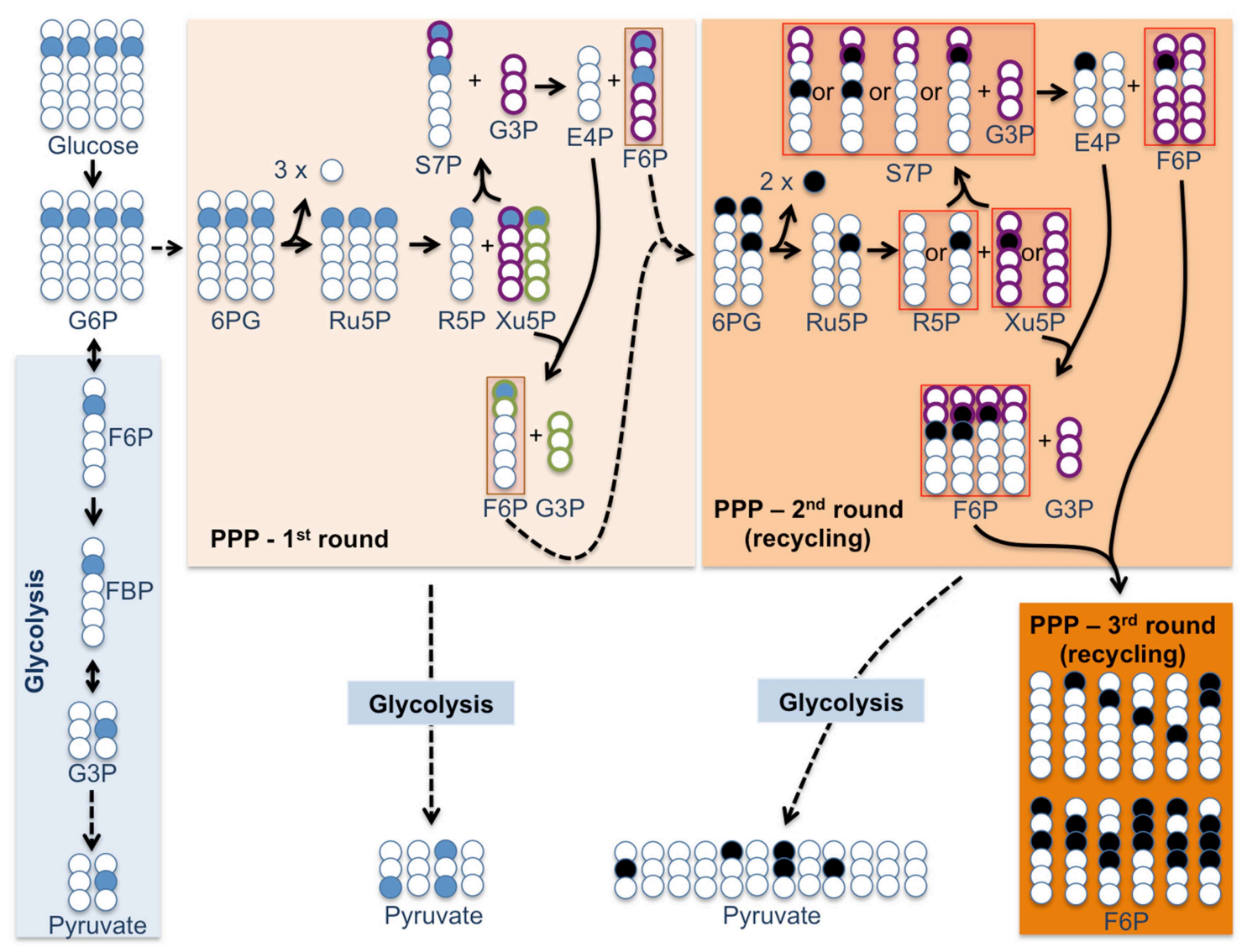

FIGURE $1 \mid{ }^{13} \mathrm{C}$-Pyruvate labeling pattern from $\left[2-{ }^{13} \mathrm{C}\right]$ glucose by recycling PPP underestimates PPP contribution to glucose metabolism. Labeling pattern in ${ }^{13} \mathrm{C}$-pyruvate originated from glycolysis ([2- $\left.{ }^{13} \mathrm{C}\right]$ pyruvate) differs from that originated from a first round of PPP ( $\left[3^{-13} \mathrm{C}\right]-$ and $\left[1,3^{-13} \mathrm{C}_{2}\right]$ pyruvate), thus helping to estimate the contribution of both pathways in glucose utilization (blue carbons). However, some cell types, such as neurons, express high PGl activity responsible for high equilibration rate of F6P with G6P, hence leading to a recycling-like PPP (PPP - second and third rounds). Identification of these recycling-PPP

${ }^{13} \mathrm{C}$-labeled intermediates (black carbons) identifies a more complex pattern of ${ }^{13} \mathrm{C}$-pyruvate labeling $\left(\left[1-{ }^{13} \mathrm{C}\right]-,\left[2-{ }^{13} \mathrm{C}\right]-\right.$, and $\left[1,2-{ }^{13} \mathrm{C}_{2}\right]$ pyruvate after the second PPP round; pattern after a third PPP round is omitted for simplicity) that overestimates glycolysis, hence underestimating the contribution of PPP to glucose utilization. Please note that $\left[1-{ }^{13} \mathrm{C}\right]$ pyruvate could also come from TCA cycle (pyruvate re-recycling; see text). However, this formation represents as low as one-twelfth of the pyruvate originated from the second round of PPP, thus likely minimally affecting to PPP activity estimation. Therefore, labeling in ${ }^{13} \mathrm{C}-\mathrm{R} 45 \mathrm{P}$ and ${ }^{13} \mathrm{C}-\mathrm{R} 5 \mathrm{P}\left(\left[2-{ }^{13} \mathrm{C}\right]-\right.$, , $\left[1,2-{ }^{13} \mathrm{C}_{2}\right]-$, $\left[1,2,3-{ }^{13} \mathrm{C}_{3}\right]$-, etc.) after the second PPP round can be used for an accurate estimation of PPP in highly recycling cells.

\section{Radioactive PPP Assessment Ex Vivo}

Several approaches have been used to estimate the fraction of G6P that is metabolized through the PPP. For experiments performed ex vivo (cultured and freshly isolated cells, or tissue slices), radiometric ${ }^{14} \mathrm{CO}_{2}$ detection after incubation of the biological samples in the presence of $\left[1{ }^{14} \mathrm{C}\right]$ glucose, under initial velocity conditions, reports flux through the oxidative branch of the PPP. However, since $\mathrm{C}_{1}-\mathrm{G} 6 \mathrm{P}$ is also decarboxylated in the tricarboxylic acid (TCA) cycle, the ${ }^{14} \mathrm{CO}_{2}$-value, taken alone, intrinsically over-estimates PPP activity. To overcome this drawback, a parallel incubation under identical conditions needs to be performed to quantify ${ }^{14} \mathrm{CO}_{2}$ collected from [6${ }^{14} \mathrm{C}$ ]glucose, since $\mathrm{C}_{6}$-G6P is exclusively decarboxylated in the TCA after G6P is converted into pyruvate both through glycolysis and PPP. Thus, the difference in the rate of ${ }^{14} \mathrm{CO}_{2}$ produced from $\left[1-{ }^{14} \mathrm{C}\right]$ glucose and that from $\left[6-{ }^{14} \mathrm{C}\right]$ glucose represents the flux of ${ }^{14} \mathrm{CO}_{2}$ exclusively produced at the oxidative PPP branch (Hothersall et al., 1979; Larrabee, 1989). Whilst this approach is suitable for ex vivo analyses, it is technically tedious for in vivo PPP assessments, as $\mathrm{CO}_{2}$ collection in vivo is hardly quantitative.

\section{${ }^{13} \mathrm{C}$-NMR PPP Assessment In Vivo}

Several approaches have been used to assess the proportion of G6P that is metabolized through the PPP in vivo-which are also useful for ex vivo. A widely used method is essentially based on the analysis of ${ }^{13} \mathrm{C}$ abundance of certain carbonatoms in lactate upon perfusion (in vivo) or incubation (ex vivo) with $\left[2-{ }^{13} \mathrm{C}\right]$ glucose. Thus, the abundance of $\left[3-{ }^{13} \mathrm{C}\right]$ lactate is assumed to be exclusively originated via PPP metabolism, whereas the abundance of $\left[2-{ }^{13} \mathrm{C}\right]$ lactate, via glycolysis (Figure 1) 
(Brekke et al., 2012). In a slightly different approach based on the use of $\left[1,2-{ }^{13} \mathrm{C}_{2}\right]$ glucose, the abundances of $\left[3-{ }^{13} \mathrm{C}\right]$ lactate and $\left[2,3-{ }^{13} \mathrm{C}\right]$ lactate are considered to be originated via PPP and glycolysis, respectively (Jalloh et al., 2015). [1,6- ${ }^{13} \mathrm{C}_{2}, 6,6-$ ${ }^{2} \mathrm{H}_{2}$ ] glucose has also been used to measure cerebral PPP activity in vivo (Ben-Yoseph et al., 1995) on the bases that it produces $\left[3-{ }^{13} \mathrm{C}\right]$ lactate and $\left[3-{ }^{13} \mathrm{C}, 3,3-{ }^{2} \mathrm{H}_{2}\right]$ lactate through glycolysis, but $\left[3-{ }^{13} \mathrm{C}, 3,3-{ }^{2} \mathrm{H}_{2}\right]$ lactate and unlabeled lactate through PPP (Ross et al., 1994). The ratios of these lactate isotopomers can be quantified using gas chromatography/mass spectrometry for calculation of PPP activity, and expressed as the percentage of glucose metabolized to lactate that passed through the PPP (Ross et al., 1994; Ben-Yoseph et al., 1995). These analyses provide information on the relative contribution of a metabolic pathway within overall glucose utilization, in contrast to the initial velocity analyses, which informs on absolute metabolic flux values. Whilst such a difference might account for the controversies in PPP activity values reported by different laboratories (HerreroMendez et al., 2009; Brekke et al., 2012; Rodriguez-Rodriguez et al., 2013), in our opinion there are some methodological concerns, as explained below.

\section{Occurrence of Recycling PPP}

A critical point that is often overlooked is the occurrence of G6P recycling from PPP-derived F6P. Thus, phosphoglucose isomerase (PGI) is a near-equilibrium enzyme that has been shown to be highly active at converting F6P into G6P in certain cells and/or tissues, such as, e.g., neurons (Gaitonde et al., 1989), which can re-enter the PPP (Figure 1). In such recycling-like $\mathrm{PPP}$, only the $\mathrm{PPP}$-derived G3P fully escapes from this cycle to be transformed into pyruvate trough glycolysis. High recovery of G6P from PPP-derived F6P may represent a bioenergetics advantage, since the redox energy of glucose can be conserved as $\mathrm{NADPH}\left(\mathrm{H}^{+}\right)$at the expense of only one carbon $\left(\mathrm{C}_{1}\right)$ per G6P. It should be noted that, in certain cells such as neurons, 6-phosphofructo-1-kinase (PFK1, which converts F6P into fructose-1,6-bisphosphate or F16BP) represents a glycolysis bottleneck (Almeida et al., 2004). Thus, PFK1 in situ activity is very low in neurons when compared, e.g., with neighbor astrocytes (Almeida et al., 2004). Such a low PFK1 activity is due to the virtual absence of 6-phosphofructo-2-kinase/fructose2,6-bisphosphatase-3 (PFKFB3) (Herrero-Mendez et al., 2009), responsible for the formation of fructose-2,6-bisphosphate (F26BP) - the most potent positive PFK1 effector. In contrast, astrocytes abundantly express PFKFB3 protein-and F26BP concentration-and, accordingly, a high in situ PFK1 activity (Almeida et al., 2004). It is therefore likely that, whereas PPPderived F6P is preferentially recycled in the PPP in neurons, it is preferably converted into lactate in astrocytes. In fact, the in situ PFK1 activity is $\sim$ four-fold lower in neurons when compared with astrocytes (Almeida et al., 2004). Therefore, we hypothesize that, in neurons, (i) most PPP-derived F6P is recycled back into the PPP, and (ii) the vast majority of G3P pool is originated directly from PPP activity. However, in astrocytes, G3P pool is originated from (i) G6P through glycolysis, (ii) PPP-derived F6P then via glycolysis, and (iii) directly from PPP activity. On our opinion, these considerations may have implications when interpreting the ${ }^{13} \mathrm{C}$-labeling data for the estimation of PPP activity, as indicated below.

\section{PPP Assessment is Intrinsically Underestimated}

When $\left[2-{ }^{13} \mathrm{C}\right]$ glucose infusion and/or incubation is used, [1$\left.{ }^{13} \mathrm{C}\right]$ - and $\left[1,3-{ }^{13} \mathrm{C}_{2}\right]$ lactate abundances are exclusively PPPderived, whereas $\left[2-{ }^{13} \mathrm{C}\right]$ lactate abundance is glycolysis-derived (Figure 1). Therefore, the relative abundance of $\left[1-{ }^{13} \mathrm{C}\right]-$ and $\left[1,3-{ }^{13} \mathrm{C}_{2}\right]$ lactate when compared with total lactate is often considered to be the proportion of glucose metabolized through the PPP (Brekke et al., 2012). However, $\left[1,3-{ }^{13} \mathrm{C}_{2}\right] \mathrm{F} 6 \mathrm{P}$, formed in the PPP from $\left[2-{ }^{13} \mathrm{C}\right]$ glucose, when converted into $[1,3-$ ${ }^{13} \mathrm{C}_{2}$ ] G6P via PGI activity (F6P $\longrightarrow$ G6P-forming), which is very high in neurons (Gaitonde et al., 1989), can produce $\left[2-{ }^{13} \mathrm{C}\right] \mathrm{F} 6 \mathrm{P}$ in the second PPP round (Figure 1). Thus, as from the second PPP round and thereafter, such $\left[2-{ }^{13} \mathrm{C}\right] \mathrm{F} 6 \mathrm{P}$ will also yield [2${ }^{13} \mathrm{C}$ lactate, largely over-estimating glycolysis. In addition, the degree of recycling PPP activity is not addressed when measuring $\left[3-{ }^{13} \mathrm{C}, 3,3-{ }^{2} \mathrm{H}_{2}\right]$ lactate plus unlabeled lactate that is formed from $\left[1,6-{ }^{13} \mathrm{C}_{2}, 6,6-{ }^{2} \mathrm{H}_{2}\right.$ ]glucose (Ben-Yoseph et al., 1995), since this approach does not address the proportion of, and rate at which F6P is recycled back into the PPP. It may therefore be concluded that the methodological approaches to estimate recycling PPP activity may be intrinsically underestimated in neurons. We hypothesize that the degree of recycling PPP activity would be best indicated by the levels of PPP-specific intermediates (Figure 1). In view that $\left[1,2-{ }^{13} \mathrm{C}_{2}\right] \mathrm{Ru} 5 \mathrm{P}$ is exclusively formed by recycling PPP from $\left[2-{ }^{13} \mathrm{C}\right]$ glucose when entering the third round, and $\left[1,2,3-{ }^{13} \mathrm{C}_{3}\right] \mathrm{Ru} 5 \mathrm{P}$ when entering the fourth round, we propose to determine the relative abundance of mono-labeled vs. multiple labeled ${ }^{13} \mathrm{C}$ - R5 P $+{ }^{13} \mathrm{C}$ - Ru5P, which is feasible by liquid chromatography/mass spectroscopy (LC/MS).

\section{Overestimation of Glycolysis}

It is a widely held custom to determine total lactate released from cells as an index of glycolysis. However, given that-at least under several circumstances-the precise proportion of G6P that is metabolized through PPP to lactate is uncertain, total lactate as an index of glycolysis should be taken with caution. We rather propose that lactate release should be considered as an overall index for glucose utilization-i.e., glycolysis plus PPP. To estimate glycolysis, the use of $\left[5-{ }^{3} \mathrm{H}\right]$ glucose conversion into ${ }^{3} \mathrm{H}_{2} \mathrm{O}$, which takes place at enolase, is often used to assess the rate of glycolysis (Neely et al., 1972). However, since enolase cannot distinguish the origin-glycolysis or PPP-of its substrate-2-phosphoglycerate-, the production of ${ }^{3} \mathrm{H}_{2} \mathrm{O}$ from $\left[5-{ }^{3} \mathrm{H}\right]$ glucose does not help to clarify the specific contribution of glycolysis to glucose utilization. Thus, the use of $\left[5-{ }^{3} \mathrm{H}\right]$ glucose could greatly overestimate the rate of glycolysis in neurons, as it has been shown in the rat heart (Goodwin et al., 2001), which in turn underestimates PPP. We encourage the use of $\left[3-{ }^{3} \mathrm{H}\right]$ glucose to estimate glycolysis, since ${ }^{3} \mathrm{H}$ of $\mathrm{C}_{3}$-glucose 
interchanges with water at aldolase (Katz et al., 1965), hence reducing the contribution of PPP to the collected ${ }^{3} \mathrm{H}_{2} \mathrm{O}$. Since PFK1 in situ activity is very low in neurons (Herrero-Mendez et al., 2009), the $\left[3-{ }^{3} \mathrm{H}\right]$ glucose approach reflects more accurately glycolysis than the $\left[5-{ }^{3} \mathrm{H}\right]$ glucose one. Using $\left[3-{ }^{3} \mathrm{H}\right]$ glucose, we have reported that primary cortical neurons produce ${ }^{3} \mathrm{H}_{2} \mathrm{O}$ at a rate that is $\sim$ four-fold lower than in primary cortical astrocytes (Almeida et al., 2004). However, the proportion of glucose that is metabolized through glycolysis in neurons can be overestimated. It has been widely reported that $\left[1-{ }^{13} \mathrm{C}\right]$ glucose perfusion in rodents renders, in the brain, a higher ${ }^{13} \mathrm{C}$-specific enrichment in $\left[4^{-13} \mathrm{C}\right]$ glutamate (mainly present in neurons) compared to [4${ }^{13} \mathrm{C}$ ]glutamine (mainly present in astrocytes) (Fitzpatrick et al., 1990; Kanamatsu and Tsukada, 1994; Bouzier et al., 1999). When comparing the specific enrichment of $\left[4{ }^{13} \mathrm{C}\right]$ glutamate and [1${ }^{13} \mathrm{C}$ ]glucose, it was calculated that $\sim 90 \%$ of the $\left[4-{ }^{13} \mathrm{C}\right]$ glutamate was originated from the glycolytic metabolism of $\left[1-{ }^{13} \mathrm{C}\right]$ glucose. The $\sim 10 \%$ loss in specific enrichment of $\left[4-{ }^{13} \mathrm{C}\right]$ glutamate is usually attributed to a loss of ${ }^{13} \mathrm{C}_{1}$ in the PPP. Unfortunately, this interpretation does not take into account that $\left[3-{ }^{13} \mathrm{C}\right]$ lactate can be actively used by neurons and converted into $\left[4-{ }^{13} \mathrm{C}\right]$ glutamate in vivo (Bouzier et al., 2000), since glycolytically-generated lactate is shuttled from astrocytes to neurons (Pellerin and Magistretti, 1994; Bouzier-Sore et al., 2006), therefore underestimating in vivo neuronal PPP.

\section{Pyruvate Re-Cycling}

Pyruvate can be re-generated from the TCA cycle intermediates malate and oxaloacetate through ME and PEPCK, respectively. Such a pyruvate re-cycling has been shown to occur in neurons (Cerdan et al., 1990; Cruz et al., 1998), where malic enzyme is expressed profusely (Vogel et al., 1998; McKenna et al., 2000), although its occurrence in astrocytes has also been proposed (Olstad et al., 2007). Therefore, when using $\left[2-{ }^{13} \mathrm{C}\right]$ glucose to estimate the PPP activity, $\left[2-{ }^{13} \mathrm{C}\right]$ pyruvate entering the TCA cycle will yield $\left[1-{ }^{13} \mathrm{C}\right]$ pyruvate from re-cycling, which will be undistinguishable from that returning from the second PPP round (Figure 1). This further reinforces our proposal that PPP should be estimated by measuring ${ }^{13} \mathrm{C}$ incorporation into the five carbon-atom sugars, instead of that into lactate or pyruvate.

\section{PPP is an Advantage for Neurons that May Failure in Neurodegeneration and Aging}

Neurons are deficient in antioxidant glutathione (Bolaños et al., 1995), hence recycling of reduced glutathione (GSH) from

\section{References}

Almeida, A., Moncada, S., and Bolaños, J. P. (2004). Nitric oxide switches on glycolysis through the AMP protein kinase and 6-phosphofructo-2-kinase pathway. Nat. Cell Biol. 6, 45-51. doi: 10.1038/ncb1080

Ben-Yoseph, O., Camp, D. M., Robinson, T. E., and Ross, B. D. (1995). Dynamic measurements of cerebral pentose phosphate pathway activity in vivo using $\left[1,6-{ }^{13} \mathrm{C}_{2}, 6,6-{ }^{2} \mathrm{H}_{2}\right]$ glucose and microdialysis. J. Neurochem. 64, 1336-1342. its oxidized form (GSSG) is critical for their survival. Since $\mathrm{NADPH}\left(\mathrm{H}^{+}\right)$is essential for this process, the re-cycling version of the $\mathrm{PPP}$ - which recovers a considerable proportion of $\mathrm{G} 6 \mathrm{P}-$ explains both the low glucose consumption and the efficient GSH regenerating activity of neurons. In contrast, astrocytes express higher PPP-rate limiting step G6PD, and PPP activity, than neurons (Garcia-Nogales et al., 2003; Herrero-Mendez et al., 2009), besides higher glycolysis (Almeida et al., 2004; HerreroMendez et al., 2009). This is likely indicating that both nonrecycling PPP plus glycolysis contribute to glucose consumption in astrocytes. Accordingly, G6P recovery through re-cycling PPP in neurons represents an advantage for neuronal survival. A large body of evidence is now showing, by PET studies, decreased glucose consumption-actually reflecting glucose uptake-in the human brain during aging and neurodegeneration (Chen and Zhong, 2013). This observation has been widely interpreted as a putative cause of limited energy production in neurons. However, before we uncover the actual contribution of PPP to glucose consumption by the brain cells, particularly neurons and astrocytes, such an assertion remains uncertain. We herein opine that the decrease in glucose utilization occurring in aging and neurodegeneration, far from merely causing a bioenergetics problem, would be responsible for an oxidative damage due to failed $\mathrm{PPP}$-derived $\mathrm{NADPH}\left(\mathrm{H}^{+}\right)$regeneration in neurons. Furthermore, we also propose that the impaired glucose utilization observed in PET studies in neurodegeneration and aging reflects, mainly, reduced glucose consumption in astrocytes. This will, in turn, result in reduced lactate released for neuronal conversion into either energy or neurotransmitter glutamate. Either hypothesis, i.e., low neuronal PPP activity causing failed antioxidant capacity, low lactate supply by astrocytes, or a combination of both, may have been largely overlooked as the cause of both bioenergetics and antioxidant neuronal damage during aging and neurodegeneration. More indepth studies to overcome the methodological drawbacks herein highlighted should be undertaken in order to unveil the actual contribution of PPP to neuronal survival in vivo.

\section{Acknowledgments}

This study has received financial support from the French State in the frame of the "Investments for the future" Programme IdEx and Labex (TRAIL) Bordeaux, reference ANR-10-IDEX03-02 and ANR-10-LABX-57. JB is funded by MINECO (SAF2013-41177-R), ISCIII (RD12/0043/0021), EU-ITN (608381), NIH/NIDA (1R21DA037678-01), MECD (Programa Estatal de Promocion del Talento y su Empleabilidad en $\mathrm{I}+\mathrm{D}+\mathrm{i}$, PRX14/00387), and the European Regional Development Fund.

Bolaños, J. P., Heales, S. J., Peuchen, S., Barker, J. E., Land, J. M. and Clark, J. B. (1995). Nitric oxide-mediated mitochondrial damage: a potential neuroprotective role for glutathione. Free Radic. Biol. Med. 21, 995-1001.

Bouzier, A. K., Quesson, B., Valeins, H., Canioni, P., and Merle, M. (1999). $\left[1-{ }^{13} \mathrm{C}\right]$ glucose metabolism in the tumoral and nontumoral cerebral tissue of a glioma-bearing rat. J. Neurochem. 72, $2445-2455$ 
Bouzier, A. K., Thiaudiere, E., Biran, M., Rouland, R., Canioni, P., and Merle, M. (2000). The metabolism of $\left[3-{ }^{13} \mathrm{C}\right]$ lactate in the rat brain is specific of a pyruvate carboxylase-deprived compartment. J. Neurochem. 75, 480-486. doi: 10.1046/j. 1471-4159.2000.0750480.x

Bouzier-Sore, A. K., Voisin, P., Bouchaud, V., Bezancon, E., Franconi, J. M., and Pellerin, L. (2006). Competition between glucose and lactate as oxidative energy substrates in both neurons and astrocytes: a comparative NMR study. Eur. J. Neurosci. 24, 1687-1694. doi: 10.1111/j.1460-9568.2006.05056.x

Brekke, E. M., Walls, A. B., Schousboe, A., Waagepetersen, H. S., and Sonnewald, U. (2012). Quantitative importance of the pentose phosphate pathway determined by incorporation of ${ }^{13} \mathrm{C}$ from $\left[2-{ }^{13} \mathrm{C}\right]-$ and [3${ }^{13} \mathrm{C}$ ]glucose into TCA cycle intermediates and neurotransmitter amino acids in functionally intact neurons. J. Cereb. Blood Flow Metab. 32, 1788-1799. doi: 10.1038/jcbfm.2012.85

Cerdan, S., Künnecke, B., and Seelig, J. (1990). Cerebral metabolism of [1,2${ }^{13} \mathrm{C}_{2}$ ] acetate as detected by in vivo and in vitro ${ }^{13} \mathrm{C}$ NMR. J. Biol. Chem. 265 , 12916-12926.

Chen, Z., and Zhong, C. (2013). Decoding Alzheimer's disease from perturbed cerebral glucose metabolism: implications for diagnostic and therapeutic strategies. Progr. Neurobiol. 108, 21-43. doi: 10.1016/j.pneurobio.2013.06.004

Cruz, F., Scott, S. R., Barroso, I., Santisteban, P., and Cerdán, S. (1998). Ontogeny and cellular localization of the pyruvate recycling system in rat brain. J. Neurochem. 70, 2613-2619.

Dringen, R., Hoepken, H. H., Minich, T., and Ruedig, C. (2007). "Pentose phosphate pathway and NADPH metabolism." in Handbook of Neurochemistry and Molecular Neurobiology, Vol. 5., Neural Energy Utilization, eds G. Dienel and G. Gibson (Heidelberg: Springer), 41-62.

Fitzpatrick, S. M., Hetherington, H. P., Behar, K. L., and Shulman, R. G. (1990). The flux from glucose to glutamate in the rat brain in vivo as determined by $1 \mathrm{H}$-observed, ${ }^{13}$ C-edited NMR spectroscopy. J. Cereb. Blood Flow Metab. 10, 170-179.

Gaitonde, M. K., Murray, E., and Cunningham, V. J. (1989). Effect of 6phosphogluconate on phosphoglucose isomerase in rat brain in vitro and in vivo. J. Neurochem. 52, 1348-1352. doi: 10.1111/j.1471-4159.1989.tb09178.x

Garcia-Nogales, P., Almeida, A., and Bolaños, J. P. (2003). Peroxynitrite protects neurons against nitric oxide-mediated apoptosis. A key role for glucose6-phosphate dehydrogenase activity in neuroprotection. J. Biol. Chem. 278, 864-874. doi: 10.1074/jbc.M206835200

Goodwin, G. W., Cohen, D. M., and Taegtmeyer, H. (2001). [5- $\left.{ }^{3} \mathrm{H}\right]$ glucose overestimates glycolytic flux in isolated working rat heart: role of the pentose phosphate pathway. Am. J. Physiol. Endocrinol. Metab. 280, E502-E508.

Herrero-Mendez, A., Almeida, A., Fernandez, E., Maestre, C., Moncada, S., and Bolaños, J. P. (2009). The bioenergetic and antioxidant status of neurons is controlled by continuous degradation of a key glycolytic enzyme by APC/CCdh1. Nat. Cell Biol. 11:747-752. doi: 10.1038/ncb1881

Hothersall, J. S., Baquer, N. Z., Greenbaum, A. L., and McLean, P. (1979). Alternative pathways of glucose utilization in brain. Changes in the pattern of glucose utilization in brain during development and the effect of phenazine methosulphate on the integration of metabolic routes. Arch. Biochem. Biophys. 198, 478-492. doi: 10.1016/0003-9861(79)90522-8

Jalloh, I., Carpenter, K. L., Grice, P., Howe, D. J., Mason, A., Gallagher, C. N., et al. (2015). Glycolysis and the pentose phosphate pathway after human traumatic brain injury: microdialysis studies using 1,2-(13)C2 glucose. J. Cereb. Blood Flow Metab. 35, 111-120. doi: 10.1038/jcbfm.2014.177

Kanamatsu, T., and Tsukada, Y. (1994). Measurement of amino acid metabolism derived from $\left[1-{ }^{13} \mathrm{C}\right]$ glucose in the rat brain using ${ }^{13} \mathrm{C}$ magnetic resonance spectroscopy. Neurochem. Res. 19, 603-612.

Katz, J., Rognstad, R., and Kemp, R. G. (1965). Isotope discrimination effects in the metabolism of tritiated glucose. J. Biol. Chem. 240, PC1484-PC1486.

Larrabee, M. G. (1989). The pentose cycle (hexose monophosphate shunt). Rigorous evaluation of limits to the flux from glucose using ${ }^{14} \mathrm{CO}_{2}$ data, with applications to peripheral ganglia of chicken embryos. J. Biol. Chem. 264, 15875-15879.

McKenna, M. C., Stevenson, J. H., Huang, X., Tildon, J. T., Zielke, C. L., and Hopkins, I. B. (2000). Mitochondrial malic enzyme activity is much higher in mitochondria from cortical synaptic terminals compared with mitochondria from primary cultures of cortical neurons or cerebellar granule cells. Neurochem. Int. 36, 451-459. doi: 10.1016/S0197-0186(99) 00148-5

Neely, J. R., Denton, R. M., England, P. J., and Randle, P. J. (1972). The effects of increased heart work on the tricarboxylate cycle and its interactions with glycolysis in the perfused rat heart. Biochem. J. 128, 147-159.

Olstad, E., Olsen, G. M., Qu, H., and Sonnewald, U. (2007). Pyruvate recycling in cultured neurons from cerebellum. J. Neurosci. Res. 85, 3318-3325. doi: 10.1002/jnr.21208

Pellerin, L., and Magistretti, P. J. (1994). Glutamate uptake into astrocytes stimulates aerobic glycolysis: a mechanism coupling neuronal activity to glucose utilization. Proc. Natl. Acad. Sci. U.S.A. 91, 10625-10629.

Rodriguez-Rodriguez, P., Fernandez, E., and Bolaños, J. P. (2013). Underestimation of the pentose-phosphate pathway in intact primary neurons as revealed by metabolic flux analysis. J. Cereb. Blood Flow Metab. 33,1843-1845. doi: 10.1038/jcbfm.2013.168

Ross, B. D., Kingsley, P. B., and Ben-Yoseph, O. (1994). Measurement of pentose phosphate-pathway activity in a single incubation with $\left[1,6-{ }^{13} \mathrm{C}_{2}, 6,6\right.$ ${ }^{2} \mathrm{H}_{2}$ ] glucose. Biochem. J. 302, 31-38.

Vogel, R., Jennemann, G., Seitz, J., Wiesinger, H., and Hamprecht, B. (1998). Mitochondrial malic enzyme: purification from bovine brain, generation of an antiserum, and immunocytochemical localization in neurons of rat brain. J. Neurochem. 71, 844-852.

Wamelink, M. M., Struys, E. A., and Jakobs, C. (2008). The biochemistry, metabolism and inherited defects of the pentose phosphate pathway: a review. J. Inherit. Metab. Dis. 31, 703-717. doi: 10.1007/s10545-0081015-6

Conflict of Interest Statement: The authors declare that the research was conducted in the absence of any commercial or financial relationships that could be construed as a potential conflict of interest.

Copyright (c) 2015 Bouzier-Sore and Bolaños. This is an open-access article distributed under the terms of the Creative Commons Attribution License (CC BY). The use, distribution or reproduction in other forums is permitted, provided the original author(s) or licensor are credited and that the original publication in this journal is cited, in accordance with accepted academic practice. No use, distribution or reproduction is permitted which does not comply with these terms. 